\title{
Análisis de las estrategias didácticas e instrumentos de evaluación del aprendizaje en un entorno virtual en la educación superior profesional. Una referencia a la Universidad Andina del Cusco
}

\author{
Nery Porcel Guzmán , José Luis Gonzales Zarate², Juan José Girón \\ Gutiérrez $^{3}$, Danitza Zúñiga Hermoza ${ }^{4}$ \\ ${ }^{1}$ Universidad Andina del Cusco, Perú \\ ${ }^{2}$ Universidad Andina del Cusco, Perú \\ ${ }^{3}$ Universidad Andina del Cusco, Perú \\ ${ }^{4}$ Universidad Andina del Cusco, Perú
}

\section{Resumen}

El sistema universitario peruano viene afrontando nuevas tendencias como la deselitización de la educación superior, la deshomogenización, la desautonomización,

la desnacionalización, la despresencialización, la internacionalización, la desgratuitarización, gestación de una nueva educación, mayor conciencia ecológica, y la educación virtual. Por ello, la investigación tiene como objetivo identificar las estrategias didácticas y los instrumentos de evaluación del aprendizaje que se deben aplicar en un entorno virtual, para brindar una educación superior de calidad en la coyuntura de la pandemia del COVID-19. La investigación tiene un enfoque cualitativo con alcance descriptivo. Se ha revisado la bibliografía y normas legales vinculadas al tema de investigación; con referencia a la Universidad Andina del Cusco aplica el nuevo paradigma educativo de la Competencia integral que tiene como fundamento el modelo filosófico antropológico del paradigma trascendental que valora la persona humana en todas sus dimensiones, prevé la formación profesional, moral, espiritual y social que estimule al estudiante a actuar en orden al bien de la sociedad y de la naturaleza. Asimismo, el presente estudio propone que las estrategias didácticas pueden utilizar: secuenciación de contenidos, conjunto de actividades propuesto a los estudiantes, metodología asociada a cada una y recursos didácticos. Los instrumentos de evaluación del aprendizaje en un entorno virtual, deben centrarse en la teoría y la práctica de la calidad total, proyectándose, hacia un auténtico desarrollo integral

humano que evalúe conocimientos, actitudes y valores, habilidades y destrezas,

tomando en consideración a la personalidad del estudiante, abandonando la evaluación tradicional, que está orientada a corregir, penalizar, sancionar y calificar.

Palabras clave: educación superior profesional, estrategias didácticas, evaluación de aprendizaje, entorno virtual. 


\title{
Analysis of didactic strategies and learning assessment instruments in a virtual environment in professional higher education. A reference to the Andean University of Cusco
}

\begin{abstract}
The Peruvian university system has been facing new trends such as the de-elitization of higher education; dehomogenization; deautonomization; denationalization; depresentialization; internationalization; de-gratification; gestation of a new education; greater ecological awareness; and, virtual education; Therefore, the research aims to identify the didactic strategies and learning assessment instruments that must be applied in a virtual environment, to provide quality higher education in the context of the COVID-19 pandemic. The research has a qualitative approach with a descriptive scope. The bibliography and legal norms related to the research topic have been reviewed; With reference to the Andean University of Cusco, the new educational paradigm of Comprehensive Competence is applied, which is based on the anthropological philosophical model of the transcendental paradigm that values the human person in all its dimensions, provides for professional, moral, spiritual and social training that stimulates the student to act in order to the good of society and nature. Didactic strategies can use: content sequencing, set of activities proposed to students, methodology associated with each one, and didactic resources. The learning evaluation instruments in a virtual environment must focus on the theory and practice of total quality, projecting themselves towards an authentic integral human development that evaluates knowledge, attitudes and values, abilities and skills, taking into consideration the personality of the student, abandoning the traditional evaluation, which is aimed at correcting, penalizing, sanctioning and qualifying.
\end{abstract}

Keywords: professional higher education, didactic strategies, learning evaluation, virtual environment.

\section{Análise de estratégias didáticas e instrumentos de avaliação da aprendizagem em ambiente virtual de educação superior profissional. Uma referência à Universidade Andina de Cusco}

\section{Resumo}

O sistema universitário peruano tem enfrentado novas tendências, como a deselitização do ensino superior; desomogenização; deseautonomização; desnacionalização; despresentialização; internacionalização; desgratificação; gestação de uma nova educação; maior consciência ecológica; e educação virtual; Portanto, o objetivo da pesquisa é identificar as estratégias didáticas e os instrumentos de avaliação da aprendizagem que devem ser aplicados em ambiente virtual, para oferecer um ensino superior de qualidade no contexto da pandemia COVID-19. A pesquisa possui abordagem qualitativa com abrangência descritiva. A bibliografia e as normas legais relacionadas ao tema de pesquisa foram revisadas; No que se refere à Universidade 
Análisis de las estrategias didácticas e instrumentos de evaluación del aprendizaje en un entorno virtual en la educación superior profesional. Una referencia a la Universidad Andina del Cusco

Andina de Cusco, aplica-se o novo paradigma educacional de Competência Integral
que se baseia no modelo filosófico antropológico do paradigma transcendental que
valoriza a pessoa humana em todas as suas dimensões, prevê profissional, moral,
espiritual e formação social que estimula o aluno a atuar em prol do bem da sociedade
e da natureza. As estratégias didáticas podem utilizar: sequenciamento de conteúdo,
conjunto de atividades propostas aos alunos, metodologia associada a cada uma e
recursos didáticos. Os instrumentos de avaliação da aprendizagem em ambiente
virtual devem ter como foco a teoria e a prática da qualidade total, projetando-se para
um autêntico desenvolvimento humano integral que avalie conhecimentos, atitudes
e valores, habilidades e competências, levando em consideração a personalidade
do aluno. abandonando a avaliação tradicional, que visa corrigir, punir, sancionar e
qualificar.

Palavras-chave: ensino superior profissional, estratégias didáticas, avaliação da aprendizagem, ambiente virtual.

\section{Introducción}

Según Rama (2014), el sistema universitario peruano y en general, de toda Latinoamérica, viene afrontando cambios con diferentes matices ante los nuevos escenarios y tendencias de la educación superior universitaria, que las denomina las macro tendencias y macro tensiones políticas. Estas nuevas tendencias serían la creciente deselitización de la educación superior, la deshomogenización, la desautonomización, la desnacionalización, la despresencialización, la internacionalización, la desgratuitarización, gestación de una nueva educación, mayor conciencia ecológica, y la educación virtual.

Es importante tener en cuenta que la educación virtual se relaciona con la educación a distancia, la cual nació a raíz de la necesidad de cobertura de calidad educativa a personas que, por distancia y tiempo, no pueden desplazarse hacia un centro de formación físico. Es así que el volumen de recursos educativos digitales impone nuevas exigencias a los sistemas e instituciones de la educación superior, en lo relativo al desarrollo de los programas educativos, los planes de estudio y los procesos de aprendizaje novedosos e innovadores, así como a las vías de acceso a la enseñanza superior.

Todo lo mencionado, propiciado por la existencia de modelos de prestación de servicios de aprendizaje en línea, a distancia, de educación abierta, mixta y cursos de corta duración, basados en la adquisición de competencias, tales como los cursos masivos en línea y de libre acceso (MOOC, por sus siglas en inglés) y los Recursos Educativos Abiertos (REA). El enorme potencial que propicia el aprendizaje en línea en general, y en particular, bajo la forma de MOOC, abre nuevas vías de acceso a la enseñanza superior, así como al aumento de las posibilidades de educación mediante soluciones alternativas flexibles. 
De acuerdo a la Resolución Viceministerial º 00093-2020-MINEDU (2020), se define al aprendizaje virtual como la adquisición de conocimientos y/o desarrollo de competencias, obtenidas y evidenciadas a través de la adaptación no presencial de los cursos, considerando tres ejes. El primero de ellos, la interacción, que es un proceso simultáneo o diferido de interaprendizaje entre docente-estudiante y estudiante-compañeros; el segundo, colaboración, consiste en acciones conjuntas facilitadas por medios tecnológicos que permiten desarrollar competencias de trabajo en equipo; el tercero, producción, comprende experiencias diseñadas por el docente y/o estudiante, a partir del uso de las herramientas de la plataforma virtual, que permitan generar evidencias de aprendizaje (Resolución Viceministerial $\mathrm{N}^{\circ}$ 00093-2020-MINEDU, 2020).

En este contexto, se deben identificar las estrategias adoptadas por las universidades peruanas al momento de adaptarse a una modalidad virtual. Esto permitirá corregir y fortalecer las estrategias de enseñanza, evaluación y aprendizaje utilizadas en contexto virtual dado por la COVID-19.

En este sentido, la investigación tiene como objetivo general, identificar las estrategias didácticas y los instrumentos de evaluación del aprendizaje que se deben aplicar en un entorno virtual para la formación profesional en las universidades peruanas.

\section{Antecedentes vinculados a la problemática de la investigación}

Para la presente investigación, trabajos como los de Salgado, con su tesis La enseñanza y el aprendizaje en modalidad virtual desde la experiencia de estudiantes y profesores de posgrado (2015), serán útiles pues suponen un antecedente. Dicha investigación tuvo por objetivo explorar las experiencias de estudiantes y profesores en un programa de posgrado de modalidad virtual, el diálogo que se establece entre estudiantes y docentes, sus formas de aprender y enseñar, así como las necesidades de apoyo en esta modalidad educativa. El trabajo de campo se llevó a cabo con estudiantes y profesores de las maestrías en Administración de Empresas y Gerencia de Proyectos, de la Facultad de Ciencias Empresariales de la Universidad Latinoamericana de Ciencia y Tecnología (ULACIT), ubicada en San José, Costa Rica, durante el tercer trimestre de 2014.

Dicha tesis planteó un estudio exploratorio y de tipo cualitativo, basado en el enfoque de la teoría fundamentada. Los datos se obtuvieron mediante grupos focales, entrevistas y el análisis de un cuestionario institucional de evaluación de los cursos respondido por los estudiantes. Los resultados se organizaron de acuerdo con seis categorías de análisis para los estudiantes, y se identificó que los estudiantes demostraron en general un nivel adecuado de satisfacción con los cursos virtuales, en un grado equivalente a los cursos presenciales de la Facultad y de la Universidad como un todo.

En el ámbito nacional, se encuentra la tesis de Vásquez titulada Aplicación de técnicas didácticas para mejorar el aprendizaje de los estudiantes de Historia Regional, de la 
Análisis de las estrategias didácticas e instrumentos de evaluación del aprendizaje en un entorno virtual en la educación superior profesional. Una referencia a la Universidad Andina del Cusco

Facultad de Ciencias Sociales U.N.S.C.H. Ayacucho 2012-II (2017). El autor estableció como objetivo general: "Determinar la mejora del aprendizaje de los estudiantes de Historia Regional, de la Facultad de Ciencias Sociales de la UNSCH 2012-II con la aplicación de las técnicas didácticas" (Vásquez, 2017, p. 21), para lo cual, el diseño elegido fue el cuasi experimental con un grupo experimental y un grupo de control, con el fin de determinar la mejora del rendimiento académico.

Entre las principales conclusiones se menciona que la aplicación de técnicas didácticas mejora significativamente el aprendizaje de los estudiantes y se recomienda capacitar a los docentes en el manejo de técnicas didácticas y hacerlos partícipes de la mejora de los aprendizajes de sus estudiantes en las diferentes asignaturas (Vásquez, 2017).

\section{Bases legales de la educación superior profesional}

Con la vigencia de Ley Universitaria, Ley № 30220, se crea la Superintendencia Nacional de Educación Superior Universitaria (Sunedu), la cual, entre sus funciones tiene la supervisión del licenciamiento de las universidades públicas y privadas en el país, y verificar el cumplimiento de la Condiciones Básicas de Calidad que estas brindan (2014). Desde su creación, esta institución ha llevado a cabo procesos de verificación de cumplimiento de la calidad de la educación brindada por las universidades, con lo cual, al año 2020, se han licenciado 94 universidades y se ha denegado la licencia a un total de 47 universidades (Sunedu, 2020). El total de universidades en funcionamiento a la fecha de promulgación de la Ley Universitaria ascendía a 140, entre públicas y privadas.

La Sunedu, ante la declaratoria de emergencia sanitaria adoptada por el Poder Ejecutivo para afrontar la epidemia del Covid-19, mediante el Decreto Supremo № 044-2020-PCM y el Decreto de Urgencia № 026-2020, emitió la Resolución del Consejo Directivo № 039-2020-SUNEDU-CD, de fecha 27 de marzo del 2020, en la cual se establecen los "Criterios para la supervisión de la adaptación de la educación no presencial, con carácter excepcional, de las asignaturas por parte de universidades y escuelas de posgrado como consecuencia de las medidas para prevenir y controlar el COVID-19" (Sunedu, 2020). Las instituciones de educación superior o técnico productivo, tal como institutos, escuelas superiores, universidades o CETPRO, deben tomar en cuenta las medidas dictadas por el Ministerio de Educación para brindar los servicios educativos, asegurando la protección contra el coronavirus (COVID-19). Todas estas medidas fueron aprobadas mediante la Resolución Viceministerial $\mathrm{N}^{\circ}$ 80-2020-MINEDU y la Resolución Viceministerial Nº 81-2020-MINEDU, en el marco del Estado de Emergencia Nacional aprobado por Decretos Supremos No 044-2020, № 051-2020-PCM, No 053-2020-PCM, № 094-2020-PCM, № 110-2020-PCM y No 1162020-PCM.

Asimismo, se emitió la Resolución Viceministerial N 085-2020-MINEDU, mediante la cual se aprobó las "Orientaciones para la continuidad del servicio educativo superior universitario, en el marco de la emergencia sanitaria, a nivel nacional" (2020). En este 
Nery Porcel Guzmán, José Luis Gonzales Zarate, Juan José Girón Gutiérrez, Danitza Zúñiga Hermoza

documento normativo se dispone de un glosario de términos que se utilizarán en el desarrollo del trabajo de investigación.

\section{Bases Teóricas vinculadas con el proceso de enseñanza aprendizaje}

\subsection{Nuevo paradigma educativo de la "competencia integral"}

El nuevo paradigma educativo de la "competencia integral" tiene como fundamento el modelo filosófico antropológico del paradigma trascendental, porque valora la persona humana en todas sus dimensiones y la pone como fin del proceso de formación, prevé no solo la formación profesional, sino también una formación moral, espiritual y social que estimule al estudiante a actuar en orden al bien de la sociedad y de la naturaleza.

Este paradigma está centrado en la formación basada en las capacidades y las competencias, que denominamos "competencia integral", entendida como un enfoque holístico en la medida en que integra y relaciona atributos y tareas, permite que ocurran varias acciones intencionales simultáneamente y toma en cuenta el contexto y la cultura del lugar donde se realiza la acción educativa y del trabajo, lo que nos permite incorporar la ética y los valores como elementos del desempeño competente. Puede decirse que en torno a las competencias se puede dar una formación integral, debido a que incluye conocimientos, potencialidades, habilidades, destrezas, prácticas, y acciones de diversa índole (personales, colectivas, afectivas, sociales y culturales) en los diferentes escenarios de aprendizaje y desempeño laboral.

El nuevo paradigma plantea formar al estudiante no solo como un competente profesional, para introducirlo en el mundo laboral, integrando el saber, saber hacer y saber actuar, sino también, incorporando las actitudes que preparan a los estudiantes para: saber ser, saber vivir y saber convivir con los demás en orden a los valores. Por ende, el estudiante no solo se forma en aptitudes profesionales, sino también en actitudes morales y axiológicas. Así, el contenido de la educación debe estar constituido por el conjunto de capacidades y competencias conformados por conocimientos, procedimientos, habilidades profesionales científico-tecnológicas, investigativas y humanísticas, que capacite al graduado para continuar su formación a lo largo de la vida con responsabilidad social y respeto al medio ambiente.

El concepto de competencia nace entre los años 80 y 90, al principio fue introducido en escuelas e institutos técnicos profesionales y posteriormente se expandió por todo el sistema educativo y estuvo basado en cuatro pilares: aprender a conocer, aprender a hacer, aprender a vivir y aprender a ser. Estos pilares se ajustan a los cambios generados por la globalización, que demanda una formación profesional por competencias que capacite al individuo para hacer frente a situaciones, trabajar en equipo y adaptarse a la flexibilidad del trabajo. Este enfoque excluye de la agenda 
educativa la formación ética axiológica que promueve la realización del hombre, desvalora la transmisión académica del conocimiento y la tradición cultural que tiene cada país.

\subsection{Principios del accionar educativo}

De acuerdo a Alcázar Gamarra (2002), la formación profesional debe tener determinadas características, las cuales se detallan a continuación:

\section{a. Integral}

La formación profesional debe estar dirigida al desarrollo de todas las dimensiones de la vida del estudiante, teniendo en cuenta su estructura constituida por una realidad bio-psico-social y trascendental. La sociedad toma cada vez mayor conciencia de la importancia que tienen los rasgos personales en el quehacer profesional. La universidad debe educar a la persona en su integridad; cada una de las asignaturas y actividades que se diseñen, tendrá que estar concebida como oportunidad para educar la inteligencia y el carácter de los estudiantes.

\section{b. Holística}

La formación profesional debe buscar que el estudiante tenga una visión sistémica de la realidad, lo que quiere decir que frente a ella tenga consciencia que es una unidad pero que se articulan componentes diversos. Debe eliminar la mirada parcial de la realidad ya que no denota toda la realidad. Comprende por ello la interdisciplinariedad, indispensable en su formación humana y profesional. Esta interdisciplinariedad debe ser tomada en cuenta en el diseño e implementación de la estructura curricular.

\section{c. Prospectiva}

Los estudiantes de hoy deberán tener en cuenta que se desempeñarán como profesionales en una sociedad diferente de la que existe hoy, ya que ella cambiará en muchos aspectos. Esta visión prospectiva les hará mirar hacia adelante, comprendiendo los posibles cambios en el futuro. La prospectiva implica considerar que, más que transmitir un círculo de conocimientos, debe la universidad proponerse hacer surgir interrogantes en la mente de los estudiantes. Por eso, la investigación, sus procesos, sus contenidos y sus descubrimientos deben ser una tarea inherente a la formación universitaria. En los ciclos iniciales de los estudios profesionales se dará especial importancia a la búsqueda, selección y procesamiento de datos proporcionados por los sistemas de información (investigación formativa), y más adelante conforme avance en su formación, la investigación intentará dar respuesta a problemas concretos ligados a su campo laboral. Es necesario preparar al estudiante para hacer frente a la incertidumbre y construir desde ella. 


\section{d. Centrada en el aprendizaje}

Entendido como enseñar educando, aprender educando y educándose; la formación profesional está centrada en el aprendizaje, del cual serán el educador y el educando los protagonistas. La enseñanza y el aprendizaje se entienden como un proceso de interacción, como una relación mutua de educador y educando, así como de educandos entre sí. Así, los contenidos de la enseñanza y los procesos, procedimientos y resultados del aprendizaje cobran relevancia.

\section{e. Con niveles de excelencia}

La excelencia, en la vida profesional, supone la resolución eficaz de los problemas, la habilidad para establecer relaciones positivas, con el entorno familiar, laboral y social. Supone, también, la inserción exitosa en el mundo laboral, además de la participación en la comunidad científica aportando de ese modo, al crecimiento del saber y su aplicación en el desarrollo de la sociedad. Su parámetro más profundo es la trascendentalidad (en el sentido de cualidades universales, como los "valores" que trascienden y van más allá de los aspectos particulares o individuales), en el que la perfección es la vocación del hombre, que se expresa en la vida práctica por la Ley de la Perfectibilidad en su sentido del aquí y del ahora y en sentido de su plenitud y desarrollo integral.

\section{f. Insertada en el mundo laboral}

La formación profesional por darse en un lugar y en un tiempo determinado, necesariamente, debe situarse en un contexto de globalización de las sociedades; reconocer y valorar su identidad nacional, seguir el avance científico y tecnológico y conocer el mercado laboral y sus proyecciones. Esta realidad exige al profesional, estar en continuo aprendizaje para responder a las nuevas exigencias de la sociedad. En el caso de la universidad, ella debe, por requerimiento de esta sociedad, examinar periódicamente las profesiones que demanda, para dar respuestas apropiadas a las necesidades del mundo laboral y social. Es necesaria también la revisión continua de los perfiles profesionales con el fin de ser actualizados.

\section{g. Comprometida con la sociedad}

La formación profesional del estudiante debe ser de excelencia por ser exigencia de la sociedad, y cuando egrese de la universidad debe retribuir con su aporte innovador a la sociedad que lo formó (Alcázar, 2002, p. 118)

\subsection{Principios que orientan la formación profesional en la Universidad Andina del Cusco}

La Universidad Andina del Cusco (1984) ha fijado una serie de principios que tienen como objetivo orientar la formación profesional en las distintas escuelas que la 
integran y que deben ser el referente para el desempeño docente y para el proceso de enseñanza-aprendizaje. A continuación, se describe cada uno de estos principios según como se señala en la Resolución № 059-CU-2016 UAC:

a. Respeto a la dignidad de la persona humana y a los derechos humanos. La educación está centrada en la condición humana.

b. En la formación profesional tiene en cuenta la estructura integral del hombre bio-psico-social y trascendental.

c. Defiende la autonomía universitaria con transparencia, rendición de cuentas y responsabilidad social.

d. Formaun profesionalintegral concalidad,fundamentadaenlainvestigación científica, integrada al desarrollo tecnológico; la investigación humanística debe estar articulada al contexto sociocultural, orientada al desarrollo sostenible de la región y del país, respondiendo a las exigencias de la persona, familia y sociedad.

e. Asume y promueve los valores andinos: YACHAY (sabiduría), LLANK'AY (trabajo), MUNAY (voluntad, afecto), AYNI (reciprocidad), los valores universales ético-estéticos, la identidad andina, el principio de intra e interculturalidad en su accionar, como elementos constitutivos de la cultura organizacional universitaria.

f. Estimula la creación intelectual y artística.

g. Difunde la producción científica, intelectual, humanística, artística y cultural con respeto a la propiedad intelectual, científica, tecnológica y cultural.

h. Promueve la internacionalización y cooperación interinstitucional a nivel nacional e internacional.

i. Defiende el pluralismo ideológico, la libertad de pensamiento y de creencias, la reflexión crítica.

j. Rechaza toda forma de violencia, intolerancia, discriminación y dependencia.

k. Impulsa el desarrollo humano, el desarrollo de la tecnología y del mercado, teniendo como fin el bienestar de las personas, sin exclusión social, de modo que el ejercicio de la ciudadanía esté sobre la base del esfuerzo y la práctica de los valores explicitados en comportamientos de rectitud, paz, amor, democracia, solidaridad, reciprocidad y trabajo (2016).

\subsection{Principios pedagógicos de la Universidad Andina del Cusco}

En relación a los aspectos pedagógicos, la Universidad Andina del Cusco difunde un conjunto de principios que rigen su actuar. Estos principios son descritos en los 
siguientes párrafos.

a. La pedagogía es abierta y activa centrada en la persona como primer valor, involucrando a la comunidad universitaria, la familia, la comunidad y la sociedad.

b. Reconoce a la persona capaz de ser formada humana, profesional y espiritualmente, con cualidades axiológicas.

c. Ningún contenido de aprendizaje puede atentar contra el proceso de perfeccionamiento integral de la persona.

d. El principio del conocimiento es la educación y el proceso de aprendizaje debe favorecer al estudiante para asegurar la significatividad de lo aprendido en la realidad de la vida, frente a su vida personal y su futura responsabilidad ante la sociedad.

e. Tanto la educación como la enseñanza aprendizaje deben ser un ejercicio en doble sentido: para lograr lo que se propone y para mantenerse en lo logrado.

f. El docente universitario como mediador del aprendizaje del estudiante y generador de su desarrollo, alimenta todos los procesos que faciliten el aprendizaje en orden a la verdad y a la toma de conciencia de su naturaleza humana.

g. La evaluación debe inspirarse en la teoría y la práctica de la calidad total, proyectándose sobre todo hacia un auténtico desarrollo humano integral, del estudiante como finalidad primordial de la educación. En este contexto, la evaluación ha de ser holística, globalizadora y continua, que evalúe conocimientos, actitudes y valores, habilidades y destrezas, tomando en consideración a toda la personalidad del estudiante, abandonando la evaluación tradicional, que está orientada a corregir, penalizar, sancionar y calificar (1984).

\subsection{Concepción del Proceso Enseñanza-Aprendizaje}

El modelo educativo de la Universidad Andina del Cusco tiene establecido roles diferenciados que deben cumplir los docentes y los estudiantes, así como la definición de los métodos y medios de enseñanza-aprendizaje que se deben aplicar, los cuales se detallan a continuación.

\section{Rol del Docente de la Universidad Andina del Cusco:}

a. Participa en el proceso dinámico de recíproca relación en la interacción educador y educando, ambos asumen el rol activo y constructivo en constante relación con la comunidad, sociedad y el ecosistema. 
Análisis de las estrategias didácticas e instrumentos de evaluación del aprendizaje en un entorno virtual en la educación superior profesional. Una referencia a la Universidad Andina del Cusco

b. Es mediador en el proceso formativo, asumiendo el compromiso de formar integralmente a los educandos con enfoque intercultural y realizando las tutorías.

c. Promueve la formación científica, tecnológica, humanística, artística, deportiva y la práctica laboral.

d. Estimula en el educando la capacidad intelectiva, el pensamiento científico, el juicio crítico, la capacidad de análisis y síntesis, y la creatividad.

e. Promueve el desarrollo de la investigación científica y la tecnología en relación con el sector productivo y para la solución de los problemas de la comunidad.

f. Rescata el respeto por la vida personal, familiar, social y étnica de los estudiantes.

g. Forma al educando en el liderazgo y el emprendimiento, la conciencia ciudadana, la solidaridad, la apertura e integración.

h. Estimula la participación de los educandos en los programas de responsabilidad social universitaria, con respeto y protección del medio ambiente, para mejorar la calidad de vida de las poblaciones.

i. Promueve el respeto a la cultura andina e identidad nacional y valora a todas las etnias que integran la nación.

j. Evalúa de forma continua, holística y globalizadora los propósitos y logros de la competencia integral con criterios cualitativos y cuantitativos.

k. Demuestra respeto y cumplimiento de la Ley Universitaria, Estatuto Universitario y de las normas internas de la Universidad.

I. Defiende la legalidad, respeta la dignidad de la persona y los derechos humanos.

\section{Rol del Estudiante Universitario de la Universidad Andina del Cusco:}

a. Demuestra autonomía intelectual, pensamiento crítico y autocrítico.

b. Demuestra disciplina en el trabajo académico.

c. Participa activamente en los proyectos de investigación científica, tecnológica y producción intelectual.

d. Se desempeña con liderazgo y sabe trabajar en equipo disciplinario e interdisciplinario.

e. Participa activamente en el desarrollo institucional universitario.

f. Es una persona comprometida con su propio desarrollo personal, 
académico, científico, deportivo y cultural durante sus estudios.

g. Adquiere las competencias necesarias para dar respuesta a la problemática profesional en el campo laboral y social.

h. Tiene un comportamiento ético en su vida personal, familiar, y social.

i. Demuestra compromiso, responsabilidad social y ciudadanía con su región y el país y respeta el medio ambiente.

j. Demuestra respeto y cumplimiento de la Ley Universitaria, Estatuto Universitario y de las normas internas de la Universidad.

k. Defiende la legalidad, respeta la dignidad de la persona y los derechos humanos.

\section{Rol de los Métodos y Medios de Enseñanza Aprendizaje:}

Las metodologías del aprendizaje que se deben aplicar deben basarse en la perspectiva filosófica antropológica de la persona humana y los principios de la psicopedagogía actual. El educador debe saber elegir las metodologías y las técnicas del aprendizaje, adecuadas para que el educando partícipe activamente en el proceso educativo, desarrollándose y formándose integralmente.

Los principios del aprendizaje que se deben tener presente en el proceso son los siguientes:

a. Principio de Integralidad.

b. Principio de la Trascendentalidad.

c. Principio Metacognitivo.

d. Principio de Metacompetencia.

e. Principio de Aplicación de la Teoría a la Práctica.

f. Principio de la Eticidad.

g. Principio de Participación en la Vida Social, Cultural y Cuidado del Medioambiente (Resolución No 059-CU-2016 UAC, 2016)

En el caso de las técnicas del aprendizaje, estas deben ayudar al educando a comprender y descubrir la presencia de la constante insatisfacción que existe en él, para que lo impulse a alcanzar progresivamente el mayor grado de perfección posible en todos los actos que acomete. Sus conocimientos, su voluntad y sus actitudes no finalizarán en sí mismos, sino se trascenderán y se proyectarán hacia los valores andinos y universales y el absoluto (ley de la perfectibilidad).

Las metodologías y técnicas de aprendizaje deben ayudar al estudiante a establecer adecuada relación consigo misma y con lo absoluto, para que la lleve a un equilibrio en su afectividad, objetivo prioritario de la acción educativa (ley de la inmanencia). 
Por otro lado, los procedimientos metodológicos adecuados permiten a la persona realizar acciones en función de la sociedad, salir de sí mismo y abrirse hacia el otro, para establecer una relación continua con el prójimo, con la sociedad y con la naturaleza (ley de la trascendencia).

\subsection{Principios didácticos basados en el paradigma trascendental}

En los siguientes párrafos, se realizará una descripción de los principios didácticos basados en el paradigma transcendental.

a. Principio Ético Axiológico. Al preparar sus clases, el educador debe tener presente la visón integral de la persona del educando y de la integralidad del proceso educativo; es decir, el educador en cada hora lectiva o práctica debe relacionarse recíprocamente con el educando a nivel ético axiológico, formándolo integralmente en sus dimensiones bio-psicosocial y trascendental. El educador con el criterio de los valores, debe elegir los procedimientos didácticos más adecuados, dando ejemplo con su comportamiento.

b. El Principio de la Relación Recíproca y Complementaria de los Contenidos y Procedimientos. Los procedimientos didácticos deben prever la dinámica interrelación y complementariedad de dos componentes indispensables del proceso educativo: a) el modo de transmitir los contenidos teóricoprácticos y b) la formación de la capacidad de perfeccionar y profundizar los conocimientos, las habilidades y actitudes adquiridas. Además, las técnicas didácticas que deben ser adoptadas, no sólo deben favorecer la óptima transmisión de los contenidos, sino también deben motivar y estimular el deseo de profundizar e investigar en el campo que están estudiando.

c. Principio de Sistematización. Los contenidos teórico-prácticos deben ser impartidos con la coherencia científica y de modo sistemático, siguiendo la lógica de la asimilación, según la secuencia: todo lo siguiente se basa en el fundamento anterior; todo aquello que se puede diferenciar en partes, se ordena cuidadosamente; lo posterior se basa en lo anterior; $y$, todo lo que ya está relacionado, se debe relacionar constantemente. Los procedimientos didácticos en este caso según el carácter de los componentes curriculares pueden ser deductivos, inductivos, analíticos, sintéticos, dogmáticos, heurísticos, etc.

d. El Principio de Vinculación de la Teoría con la Práctica. Los procedimientos didácticos deben necesariamente ligar los saberes que adquiere el estudiante con la aplicación práctica de estos saberes en la vida tanto en el trabajo como en lo personal. El estudiante debe ser motivado, para comprender el porqué de su estudio.

e. El Principio de la Asequibilidad. Este requiere que la transmisión de 
Nery Porcel Guzmán, José Luis Gonzales Zarate, Juan José Girón Gutiérrez, Danitza Zúñiga Hermoza

los contenidos teórico-prácticos y formación de las actitudes sean comprensibles y adecuadas a las características personales de los educandos.

\section{f. Principio de Vinculación de la Persona con el Medio Social, Cultural y} Ecológico. Los procedimientos didácticos elegidos, deben siempre tener presente el contexto socio-cultural del estudiante. Es decir, el educador debe considerar las características propias del ambiente vital de los educandos, rasgos nacionales, los valores sociales y culturales, y ecológicos.

\subsection{Medios y materiales}

Los medios y los materiales son los elementos indispensables en el proceso educativo. La elección de los medios y materiales dependen estrictamente de las características específicas de cada asignatura, pero la utilización de dichos instrumentos es indispensable para optimizar el desarrollo del proceso de enseñanza aprendizaje. Los medios son cualquier objeto o recurso que se utiliza con la intención didáctica de articular, mediante un determinado sistema de símbolos, ciertos mensajes en orden a su aplicación con fines instructivos, los cuales se ponen al servicio de docentes y estudiantes para llevar a cabo el desarrollo curricular académico. Los materiales, según Gimeno (2008), son cualquier instrumento que pueda servir como recurso para el aprendizaje o el desarrollo de alguna función de la enseñanza. Asimismo, Castillo y Cabrerizo (2006) afirman que debido a la polisemia entre medios, materiales y recursos, se pueden utilizar indistintamente, para referirnos a todo tipo de apoyos e instrumentos utilizados al servicio del proceso de enseñanza aprendizaje, ya sean escritos, impresos, de carácter virtual o informático o a las Tecnologías de la Información y la Comunicación (TIC) aplicadas al ámbito curricular, sin olvidar que su diversidad y sus características confieren a cada uno personalidad propia, lo que les hace más idóneos para su utilización en determinados momentos y situaciones del proceso de enseñanza aprendizaje.

Para que un material didáctico resulte eficaz en el logro de los aprendizajes, no basta con que sea un buen material o la última tecnología o el más caro del mercado, se debe considerar en qué medida sus características específicas, como los contenidos y actividades, están en consonancia con los aspectos curriculares fundamentales de una asignatura específica en un contexto académico determinado. En consecuencia, para elegir los materiales se debe evaluar previamente:

a. Los objetivos educativos que pretendemos lograr utilizando el material.

b. Los contenidos que se van a trabajar utilizando el material, si están en sintonía con los contenidos de la asignatura.

c. Las características del contexto en el que se desarrolla la docencia y los recursos disponibles en donde se va a emplear el material que se está seleccionando. 
d. Las características de los estudiantes que los utilizarán, estilos cognitivos o de aprendizaje, intereses, conocimientos previos, experiencia y habilidades requeridas para el uso de los materiales.

e. Las estrategias didácticas que se pueden diseñar utilizando el material seleccionado: secuenciación de los contenidos, el conjunto de actividades que se pueden proponer a los estudiantes, la metodología asociada a cada una, los recursos didácticos que se pueden emplear, etc. El uso de un determinado recurso didáctico no debe condicionar los contenidos a tratar o la estrategia didáctica que se va a emplear.

Debido a la nueva sociedad de la información o sociedad del conocimiento asentada en el principio de globalización cultural y económica y a los constantes avances científico-tecnológicos y el cambiante entorno social, los docentes y estudiantes deben hacer uso de las tecnologías de información y comunicación como materiales curriculares habituales en el proceso de enseñanza aprendizaje. Debido a ello, las horas de dedicación pueden proyectarse más allá de las horas de docencia presencial, lo que implica nuevas necesidades de formación en estrategias metodológicas de enseñanza aprendizaje con las TIC y nuevas inversiones en tiempo y equipos informáticos.

\subsection{Marco Conceptual}

Con el objetivo de estandarizar el uso de los conceptos vinculados al tema de investigación, se presenta una serie de definiciones que ayudarán al lector para una mejor comprensión de la problemática analizada. Según la Resolución Viceministerial $\mathrm{N}^{\circ}$ 085-2020-MINEDU se tiene en cuenta la siguiente terminología:

a. Aprendizaje: proceso de asimilación de los conocimientos, por parte del estudiante. En la actualidad el aprender se extiende a toda la vida; movimientos, destrezas, sensibilidad, conducta. Proceso mediante el cual el sujeto incorpora o modifica una experiencia a su presente conocimiento o destreza. Es el motivo imprescindible del acto formativo (García, 2005, p. 289).

b. Área curricular: conjunto de conocimientos científicos y técnicos, que por su afinidad conceptual, teórica y metodológica conforman una porción claramente identificable de los contenidos de un plan de estudio en una carrera técnica, de licenciatura, o de postgrado. Por ejemplo: área de formación especializada, área de formación general área de formación instrumental (Glosario regional de América Latina sobre la Educación Superior. IESALC, p. 289).

c. Área especialidad: corresponden a las propuestas de asignaturas que ofrecen herramientas y procedimientos para la intervención profesional 
(Dirección General de Investigación y Acreditación Universitaria (2005). Modelo de autoevaluación con fines de mejora de las carreras universitarias. Lima: Asamblea Nacional de Rectores).

d. Asignatura: cada una de las materias en que se estructura un plan de estudios, aunque es posible que una materia comprenda varias asignaturas. Cada asignatura suele tener atribuidos un número de créditos determinado, de acuerdo con la dedicación de horas de docencia o de trabajo total de los estudiantes. Hay diversos tipos de asignaturas: troncales, obligatorias, optativas, de libre elección, comunes, de especialización (Red Iberoamericana para la Acreditación de la Calidad de la Educación Superior RIACES (2004). Op.Cit).

e. Aula virtual: entorno digital, plataforma virtual, plataforma educativa o entorno virtual de aprendizaje donde se realiza la locación, que incluye los contenidos, recursos y actividades de aprendizaje, además de herramientas de comunicación sincrónica y asincrónica.

f. Carrera profesional: estudios superiores que habilitan para el ejercicio de una profesión. Y está constituido por cuatro subprocesos: enseñanzaaprendizaje, investigación formativa, extensión universitaria y proyección social (DEA-CONEAU, 2008). Cambiar por Escuela Profesional.

g. Competencia: capacidad de poner en práctica de forma integrada, en contextos diferentes, los conocimientos, habilidades y características de la personalidad adquiridas y/o desarrolladas. Incluye saber teórico (saber-saber), habilidades practicas aplicativas (saber-hacer), actitudes (compromisos personales, saber-ser y saber-convivir). Laboralmente se interpretan como aquellas características de una persona que están relacionadas con una actuación exitosa en el puesto de trabajo. Las competencias pueden consistir en: motivos, rasgos de carácter, actitudes, conocimientos, habilidades y comportamientos (García Rocha, J.A. Glosario de Términos Básicos en regulación y Acreditación en Educación Superior Virtual y Transfronteriza, pág.300).

h. Crédito académico: unidad de medida para asignar el valor a un tiempo determinado de trabajo académico, de acuerdo a la naturaleza de la signatura. (Instituto de Investigación y Fomento de la modernización y acreditación universitaria (2000). ANR, Lima-Perú, Op Cit) Unidad de valor asignado a los cursos en función de su peso académico dentro de un plan de estudios. Cada curso puede valer uno o más créditos. (Raúl Estuardo C. (s.f) Manual de Terminología Universitaria. CONUP, Lima-Perú, pag.85).

i. Currículo: instrumento de planificación académica universitaria que, plasmando un modelo educativo, orienta e instrumenta el desarrollo de una carrera profesional, de acuerdo a un perfil o indicadores previamente establecidos (Instituto Internacional para la Educación Superior en América Latina y el Caribe IESALC (2007). Op.Cit). Conjunto interrelacionado de 
conceptos, políticas, lineamientos, proposiciones y estrategias educativas que norman y conducen explícitamente los procesos de enseñanzaaprendizaje, para el desarrollo y la formación integral de los estudiantes en el ámbito de la educación superior (Glosario Regional de América Latina sobre la Educación Superior. IESALC, pág. 303).

j. Docencia: función sustantiva de la universidad que implica la realización directa de los procesos de enseñanza-aprendizaje, el cual incluye el diagnostico, la planificación, la ejecución y la evaluación de los procesos formativos y sus resultados y de otras actividades educativas. La función docente, además de la asignación académica, comprende también las actividades de actualización y perfeccionamiento pedagógico; evaluación institucional (Instituto Internacional para la Educación Superior en América Latina y el Caribe IESAL (2007). Op. Cit).

k. Docente: el que enseña, el maestro, profesor o catedrático. En la universidad, es la persona dedicada a la enseñanza-aprendizaje en los niveles de pregrado, postgrado, cursos especializados o de investigación. Genéricamente se habla de personal docente. (Raúl Estuardo C. (s.f.) Manual de terminología Universitaria. CONUP, Lima-Perú, pág. 115). Es un agente del proceso educativo, tiene como misión contribuir eficazmente a la formación de los estudiantes en todas las dimensiones del desarrollo humano (Ley N² 29394-2009. Ley de Institutos y Escuelas de Educación Superior. Lima-Perú. Art. 38).

I. Emergencia sanitaria: medida excepcional de prevención y control para evitar la aparición y propagación del COVID-19, dispuesta por el Decreto Supremo $N^{\circ}$ 008-2020-SA, por un plazo de noventa (90) días calendario, contados a partir del 12 de mayo de 2020.

m. Enseñanza: acto que realiza el docente para apoyar o facilitar el aprendizaje del alumno, utilizando métodos, procedimientos, estrategias, técnicas y recursos específicos. Entre sus elementos centrales esta la experiencia y el capital cultural del docente, apoyados en la idea básica de que lo importante es propiciar aprendizajes. (Instituto Internacional para la Educación Superior en América Latina y el Caribe IESAL (2007). Op. Cit. Pag. 313).

n. Estudiante: son estudiantes universitarios quienes han aprobado el nivel de educación secundaria, han cumplido con los requisitos establecidos para su admisión en la Universidad y se han matriculado en ella (...) (ley Universitaria 23733, 1983. Lima-Perú, art. 55%).

o. Evaluación: es un instrumento de fomento de la calidad de la educación que tiene por objeto la medición de los resultados y dificultades en el cumplimiento de las metas previstas en términos de aprendizajes, destrezas y competencias comprometidos con los estudiantes, la sociedad y el Estado, así como proponer políticas, programas y acciones para el 
mejoramiento de la calidad educativa. (Ley 28740. 2006. Lima-Perú, art. $11^{\circ}$ ) Es un proceso dinámico, sistemático, riguroso, transparente, abierto y participativo, apoyado en datos, informaciones, fuentes y agentes diversos y explícitamente incorporados en el proceso de toma de decisiones.

p. Evaluación de competencias: proceso permanente que permite tomar decisiones y emitir juicios, acerca de los logros obtenidos por un participante, durante y al concluir la experiencia educativa. (García Rocha, J.A. 2005. Glosario de Términos Básicos en regulación y Acreditación en Educación Superior Virtual y Transfronteriza, pag.316).

q. Evaluación de competencias: la evaluación basada en competencias puede considerarse dentro del concepto de evaluación formativa. Por tanto, permite encontrar las brechas entre el desempeño mostrado y el desempeño requerido, y trazar planes de desarrollo con acciones de capacitación para mejorar la calidad en el desempeño. Se centra en demostrar las competencias en acción, en un desempeño profesional observable y plenamente definido. (Adaptado del SENA, Colombia y del texto de M. Irigoin y F. Vargas "Competencia Laboral” 2002. Pág. 137-138).

r. Evaluación formativa: evaluación de aprendizaje (inicial, formativa o sumativa) que se realiza a un estudiante, en un nivel cualitativo e integrando actitudes (valores), destrezas y procesamiento de la información por el alumno. (García Rocha, J.A. 2005. Glosario de Términos Básicos en regulación y Acreditación en Educación Superior Virtual y Transfronteriza, pag.316).

s. Investigación formativa: alude a la dinámica de la relación entre el conocimiento y los procesos académicos. Esto es, reconocimiento de que el proceso de aprendizaje es un proceso de construcción del conocimiento, que a enseñanza debe ser objeto de reflexión sistemática sobre la base de la vinculación entre teoría y experiencia pedagógica y que el docente debe estar comprometido también en el proceso de construcción y sistematización del saber en qué consiste la actualización permanente (Consejo Nacional de evaluación CNA. La evaluación externa en el contexto de la acreditación en Colombia).

t. Plataforma virtual o plataforma educativa: espacio en donde se imparte el servicio educativo de los cursos, basados en tecnologías de la información y comunicación, ya sea un software, una web, entre otros. Cada plataforma posee funciones propias por lo que se definirá su pertinencia de acuerdo al modelo educativo, la metodología y diseño didáctico correspondiente, según las necesidades del usuario que se trate. (Resolución Viceministerial $\mathrm{N}^{\circ}$ 085-2020-MINEDU, 2020, pp. 3-4). 
Análisis de las estrategias didácticas e instrumentos de evaluación del aprendizaje en un entorno virtual en la educación superior profesional. Una referencia a la Universidad Andina del Cusco

\section{Metodología}

La investigación tiene un enfoque cualitativo con un alcance descriptivo, debido a que busca conocer las propiedades, dimensiones y características del fenómeno que se está sometiendo a estudio, el cual está referido a las variables estrategias de enseñanza y estrategias de evaluación. Para tal efecto, se realizará una revisión general de la bibliografía vinculada al tema de investigación, así como a las normas legales relacionadas, con una referencia general a las normas que rigen el proceso de enseñanza-aprendizaje en la Universidad Andina del Cusco.

\section{Conclusiones}

Del análisis a las estrategias didácticas que se pueden emplear utilizando el material seleccionado: secuenciación de los contenidos, el conjunto de actividades que se pueden proponer a los estudiantes, la metodología asociada a cada una, los recursos didácticos que se pueden emplear, entre otros. El uso de un determinado recurso didáctico no debe condicionar los contenidos a tratar o la estrategia didáctica que se va a emplear.

Del análisis a los instrumentos de evaluación del aprendizaje en un entorno virtual, debe centrarse en la teoría y la práctica de la calidad total, proyectándose, hacia un auténtico desarrollo integral humano, del alumno como finalidad primordial de la educación. En este entender, la evaluación ha de ser holística, globalizadora y continua, que evalúe conocimientos, actitudes y valores, habilidades y destrezas, tomando en consideración a toda la personalidad del estudiante, abandonando la evaluación tradicional, que está orientada a corregir, penalizar, sancionar y calificar.

\section{Recomendaciones}

Se recomienda que las estrategias didácticas del aprendizaje en un entorno virtual en la educación superior profesional sean evaluadas permanentemente como fomento de la calidad de la educación, la cual tiene por objeto la medición de los resultados y dificultades en el cumplimiento de las metas previstas en términos de aprendizajes, destrezas y competencias comprometidos con los alumnos, la sociedad y el Estado. Asimismo, se recomienda proponer políticas, programas y acciones para el mejoramiento de la calidad educativa, así como en el proceso de toma de decisiones, lo cual permitirá evitar la deserción masiva de estudiantes.

Se recomienda que los instrumentos de evaluación de aprendizaje en un entorno virtual en la educación superior profesional estén en permanente evaluación debido al avance tecnológico y globalizado. Estos instrumentos deben estar en continuo desarrollo y proyectándose al auténtico crecimiento integral humano del alumno. 
Nery Porcel Guzmán, José Luis Gonzales Zarate, Juan José Girón Gutiérrez, Danitza Zúñiga Hermoza

\section{Referencias bibliográficas}

Alcazar Gamarra, M. (2002). Curriculum universitario para el siglo XXI. Lima: Lima Universidad nacional de educacion Enrique Guzman y Valle.

Castillo, S., \& Cabrerizo, J. (2006). Evaluación Educativa de Aprendizajes y Competencias. Madrid-España: Pearson Educación.

Gimeno, J. (2008). Educar por competencias, ¿que hay de nuevo? Valencia: Morata.

Ley N ${ }^{\circ}$ 30230. Ley Universitaria. Congreso de la República (2014). Recuperado de https://leyes.congreso.gob.pe/Documentos/Leyes/30220.pdf

Rama, C. (2014). La internacionalización de la educación superior en America Latina. México: México.

Resolución del Consejo Directivo No 039-2020-SUNEDU-CDSunedu. Criterios para la supervisión de la adaptación de la educación no presencial, con carácter excepcional, de las asignaturas por parte de universidades y escuelas de posgrado como consecuencia de las medidas para prevenir y controlar el COVID-19. SUNEDU (2020). Recuperado de https://busquedas.elperuano.pe/normaslegales/apruebanlos-criterios-para-la-supervision-de-la-adaptacion-resolucion-n-039-2020-suneducd-1865206-1/

Resolución No 059-CU-2016 UAC. Modelo Educativo de la Universidad Andina del Cusco. Universidad Andina del Cusco (2016). Recuperado de https://www.uandina. edu.pe/descargas/transparencia/copea-2016/copea-CO.pdf

Resolución Viceministerial $N^{\circ}$ 00093-2020-MINEDU. Orientaciones pedagógicas para el servicio educativo de Educación Básica durante el año 2020 en el marco de la emergencia sanitaria por el Coronavirus COVID-19. Ministerio de Educación (2020). Recuperado de https://cdn.www.gob.pe/uploads/document/file/632256/ RVM_N_093-2020-MINEDU.pdf

Resolución Viceministerial N 085-2020-MINEDU - Orientaciones para la continuidad del servicio educativo superior universitario, en el marco de la emergencia sanitaria, a nivel nacional. Ministerio de Educación (2020). Recuperado de https://cdn.www. gob.pe/uploads/document/file/574842/RVM_N_085-2020-MINEDU.pdf

Salgado, E. (2015). La enseñanza y el aprendizaje en modalidad virtual desde la experiencia de estudiantes y profesores de posgrado (Tesis de Doctorado, Universidad Católica de Costa Rica, San José, Costa Rica).

Sunedu. (2020). Universidades Licenciadas. Recuperado de https://www.sunedu.gob. pe/lista-de-universidades-licenciadas/

Universidad Andina del Cusco. (1984). Universidad Andina del Cusco. Cusco: Perú.

Vásquez, J. (2017). Aplicación de técnicas didácticas para mejorar el aprendizaje de los estudiantes de Historia Regional, de la Facultad de Ciencias Sociales U.N.S.C.H. Ayacucho 2012-II (Tesis de Doctorado, Universidad Nacional de Educación Enrique Guzmán y Valle, Lima, Perú). 
Análisis de las estrategias didácticas e instrumentos de evaluación del aprendizaje en un entorno virtual en la educación superior profesional. Una referencia a la Universidad Andina del Cusco

Fecha de recepción: 08/12/2020

Fecha de aceptación: 15/05/2021 Correspondencia:nporcel@uandina.edu.pe jgonzales@uandina.edu.pe jgiron@uandina.edu.pe dzuñiga@uandina.edu.pe 